\title{
Percepções e conhecimentos dos estudantes sobre fungos
}

\author{
Perceptions and knowledge of students about fungi
}

\author{
A. C. Silva*; A. M. S. Gouw \\ Programa de Pós-Graduação em Ensino de Ciências e Matemática/ Instituto de Ciências Ambientais, Químicas e \\ Farmacêuticas, Universidade Federal de São Paulo, 09913-030, Diadema-SP, Brasil \\ *biologaalinecosta@@gmail.com \\ (Recebido em 11 de setembro de 2020; aceito em 01 de junho de 2021)
}

\begin{abstract}
O presente estudo trata-se de uma pesquisa sobre as percepções e conhecimentos que os estudantes concluintes do ensino médio apresentam sobre fungos, organismos de importância ecológica, médica e econômica. O percurso metodológico desta investigação envolveu a participação de 305 estudantes da rede pública e privada do município de Diadema/SP, cidade localizada na Região Metropolitana do Estado de São Paulo. A coleta de dados ocorreu por meio de um questionário de pesquisa, construído e validado para compreender a visão dos estudantes sobre o assunto. Os resultados evidenciaram que os estudantes finalizam a educação básica com conhecimentos limitados sobre os fungos, de forma a pouco perceber a importância desses organismos no meio ambiente e na vida cotidiana, além de exibir concepções equivocadas sobre esses organismos. Espera-se que os resultados deste estudo ampliem o debate e auxilie em reflexões sobre o ensino de micologia nas escolas e nas instituições de formação de professores, de modo a impactar positivamente no fazer pedagógico dos professores e, consequentemente, no aprender dos estudantes, em especial, quando $o$ assunto em questão forem os fungos.

Palavras-chave: Reino Fungi, Ensino de Micologia, Ensino de Ciências.
\end{abstract}

The present study is aimed to investigate the perceptions and knowledge that high school students present about fungi, organisms of ecological, medical and economic importance. The methodological path of this investigation involved the participation of 305 students from public and private schools in Diadema, SP, a city located in the metropolitan area of the state of São Paulo. Data collection was carried out by applying a research questionnaire, developed and validated to understand the view of students on the subject. The results showed that students finish basic education with limited knowledge about fungi and barely understand the importance of these organisms in the environment and in everyday life. In addition to showing misconceptions about these organisms, it is expected that the results of this study will broaden the debates and reflections on the teaching of mycology in schools and in teacher training institutions in order to positively impact on the pedagogical practice of teachers and, consequently, on the learning of students, especially when the subject is fungi.

Keywords: Fungi Kingdom, Teaching of Mycology, Science Education.

\section{INTRODUÇÃO}

Entre a diversidade de seres vivos que compõe a teia da vida estão os fungos, organismos que desempenham papel essencial na natureza e na manutenção da vida [1]. Esses organismos apresentam destaque no âmbito desta pesquisa, pois o presente estudo teve por objetivo investigar as percepções e conhecimentos que os estudantes concluintes do ensino médio apresentam sobre tais seres vivos.

É importante salientar que apesar dos fungos terem um reconhecível papel na teia da vida e se destacarem devido a sua importância ecológica, médica e econômica [1, 2], o que torna a presença desses organismos constante em nosso cotidiano, ainda muito pouco se discute sobre os fungos na educação básica [3, 4], que é etapa na qual os estudantes têm a oportunidade de construir conhecimentos científicos sobre esses organismos na escola.

Vale salientar que os estudos sobre a abordagem dos fungos na educação básica são limitados, e a literatura científica educacional sobre o tema é escassa [4]. Há poucas pesquisas sobre o ensino de micologia nas escolas [4].

Outra questão importante é sobre a abordagem do conteúdo de fungos nas aulas escolares, que muitas das vezes é apresentado a partir de uma perspectiva exclusivamente expositiva e com pouca contextualização $[4,5]$, o que dificulta o estabelecimento de conexões entre o conteúdo e o 
cotidiano do aluno o que, consequentemente, não contribui para construção de uma alfabetização científica sobre a temática. Uma parcela significativa dos estudantes, quando finalizam a educação básica, apresentam uma visão distorcida a respeito dos fungos, associando-os, predominantemente, a organismos maléficos e causadores de doenças, exibindo pouco conhecimento da importância ecológica, médica e econômica desses seres [4], justamente em consequência dessa abordagem pouco atrativa e sem estímulo a questionamentos e discussões.

Em razão deste cenário, é pertinente a realização de pesquisas que visam promover o ensino de micologia de forma a construir alicerces que impactem os currículos escolares, a formação de professores e principalmente, as salas de aula. Espera-se que os resultados desta pesquisa fomentem e contribuam com discussões que visem melhorar a qualidade do ensino de ciências e biologia na educação básica, especialmente quando o assunto em questão forem os fungos.

\section{MATERIAL E MÉTODOS}

\subsection{Desenho Geral}

A pesquisa tratou-se de uma investigação que teve como público-alvo estudantes concluintes do ensino médio. Acredita-se que a escolha em questão possa oferecer indícios das possíveis contribuições da escola no que se refere ao ensino de micologia, já que o estudante estava prestes a finalizar a educação básica. Assim, a ideia é que os estudantes utilizassem de toda sua "bagagem escolar" (do ensino infantil, fundamental e médio) para responder a pesquisa.

Ao total, 305 estudantes participaram do estudo (rede pública e privada), que foi desenvolvido no município de Diadema, cidade localizada na Região Metropolitana do Estado de São Paulo.

\subsection{Coleta de Dados}

O instrumento de coleta de dados utilizado na investigação foi um questionário de pesquisa (Quadro 1), que foi construído e validado dentro do Grupo de Pesquisa "Perspectivas para o Conhecimento Biológico" da Universidade Federal de São Paulo - campus Diadema.

De cunho anônimo, o questionário foi composto por 29 afirmações sobre os fungos, na qual os estudantes, em uma escala do tipo Likert de quatro pontos, tinham que assinalar: (1) "Discordo Totalmente"; (2) "Discordo em Parte"; (3) "Concordo em Parte" ou (4) "Concordo Plenamente". Também foi dada a opção ao estudante de manter a afirmação em branco caso não soubesse opinar ou responder sobre o assunto.

Cabe acentuar o motivo pelo qual o questionário foi construído em escala do tipo Likert, uma escala de resposta psicométrica usada habitualmente em questionários de pesquisa de opinião [6]. Essa estratégia foi pensada para que o estudante não enxergasse o questionário como uma prova ou avaliação e, sim, apenas como um instrumento de pesquisa e coleta de opinião.

Para análise dos dados foi levado em consideração o valor 2,5, que corresponde ao valor médio da escala Likert de quatro pontos. Dessa forma, médias acima desse valor indicam concordância com a afirmação, enquanto médias abaixo desse valor indicam discordância.

A coleta de dados ocorreu diretamente nas escolas, ao final do mês de outubro/2017. O questionário foi aplicado pelos próprios pesquisadores, com permissão de algum professor titular da turma.

\subsection{Questionário de Pesquisa}

O questionário de pesquisa (Quadro 1), construído e validado para coleta de dados, foi composto por 29 afirmativas, sendo 16 conceitualmente corretas e 13 incorretas, para que o estudante utilizasse de suas percepções e conhecimentos para concordar ou discordar dessas afirmações. Todavia, neste artigo (nas próximas tabelas), para melhor compreensão dos dados, as afirmações conceitualmente incorretas serão destacadas com um asterisco (*). 
É importante esclarecer que as afirmações, apesar de aparecerem de forma de aleatória no questionário, elas fazem parte de cinco diferentes fatores, que são grupos de afirmações com a mesma temática. Esses fatores foram: Percepção Geral (A1), Saúde e Doença (A05; A06; A11; A12; A21; A24; A25; A26), Ciência Tecnologia e Sociedade (A03; A08; A14; A17; A20; A28), Ecologia e Ambiente (A04; A16; A22; A23;A29) e Biologia dos Fungos (A02; A07; A09; A10; A13; A15; A18; A19; A27).

Quadro 1: Questionário de Pesquisa sobre percepções e conhecimentos dos estudantes sobre fungos.

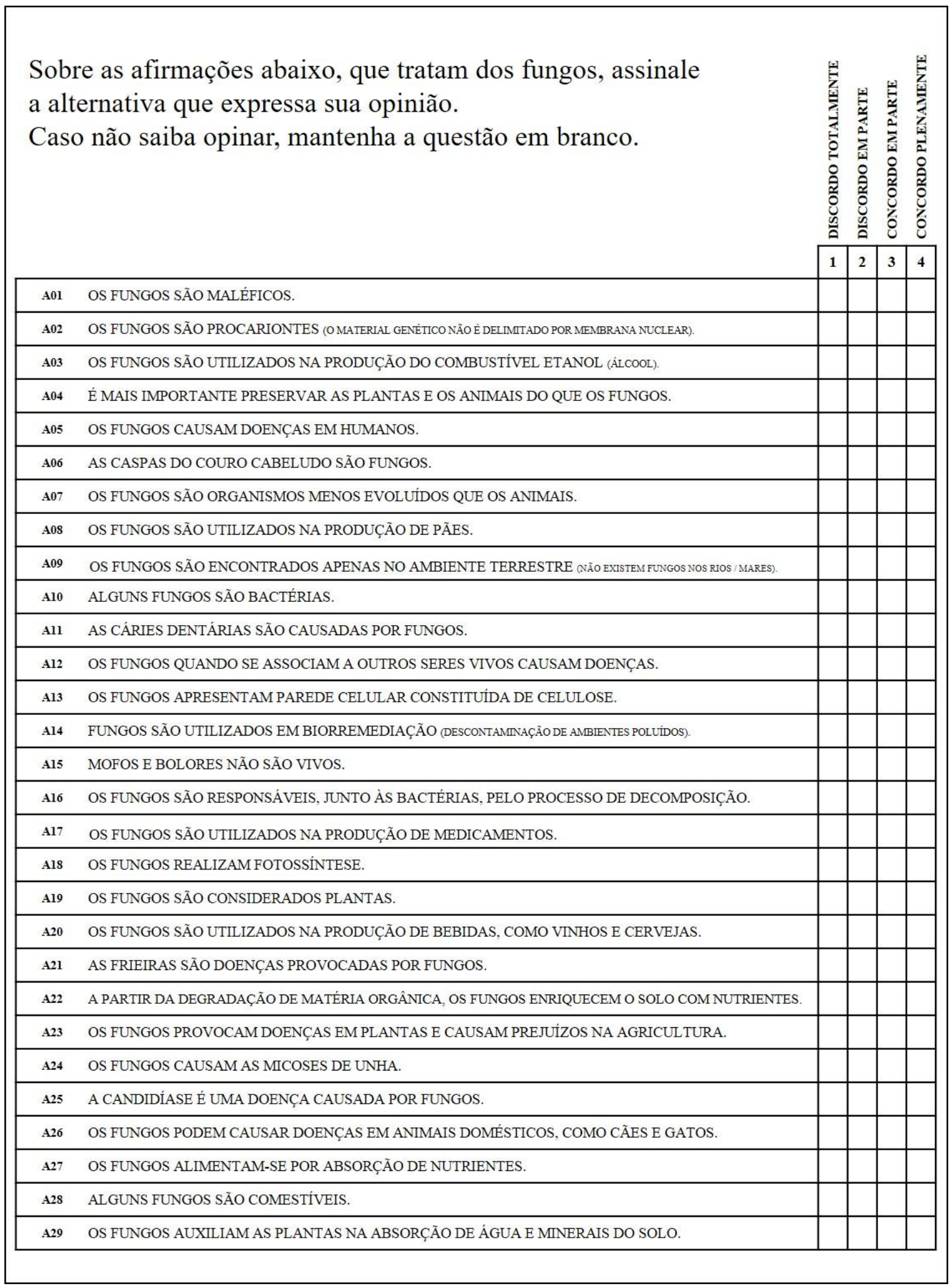




\subsection{Aspectos Éticos da Pesquisa}

Todas as etapas da pesquisa foram realizadas respeitando-se as recomendações do Comitê de Ética em Pesquisa (CEP) da Universidade Federal de São Paulo, sendo o projeto aprovado em 08/06/2017 ( $\mathrm{N}^{\mathrm{o}}$ 0425/2017).

Por se tratar de um estudo que envolveu pesquisa diretamente com seres humanos, toda comunidade escolar envolvida (alunos, pais e/ou responsáveis e diretores escolares) consentiu voluntariamente a participação na pesquisa por meio de assinatura de Termo de Consentimento Livre e Esclarecido e termo de assentimento.

\subsection{Características da Pesquisa}

A pesquisa foi caracterizada como um estudo exploratório, que é um tipo de pesquisa que busca levantar informações sobre um determinado objeto que geralmente foi pouco explorado no meio científico [7].

Já a amostra investigada $(n=305)$ foi dada por conveniência, um tipo de amostra nãoprobabilística, que é estimada a partir de critérios julgados pelos pesquisadores, que admite que o número estimado possa representar um universo [8].

Quanto a natureza da pesquisa, esta foi classificada como predominantemente quantitativa, pois recorreu à uma linguagem matemática para organizar, analisar e interpretar dados a respeito do fenômeno [9], ou seja, a percepção e o conhecimento dos estudantes sobre fungos.

\section{RESULTADOS E DISCUSSÃO}

Com base nas médias das respostas dos participantes da pesquisa, foi possível evidenciar a percepção e o conhecimento que os estudantes possuem sobre os conteúdos micológicos quando finalizam a educação básica. $\mathrm{O}$ valor de 2,5 corresponde ao valor médio da escala Likert de quatro pontos, assim, médias acima desse valor indicaram concordância dos estudantes com a afirmação, enquanto médias abaixo desse valor indicaram discordância.

\subsection{Fator "Percepção Geral"}

Em relação a análise da única afirmação do Fator "Percepção Geral" (Tabela 1), esta sinaliza que os estudantes tendem a uma percepção de que os fungos são organismos maléficos (Q3 > 2,5).

Tabela 1: Média da afirmação do fator "Percepção Geral".

\begin{tabular}{clcccc}
\hline Afirmação & Descrição & (n) & Média & DP \\
\hline *A 01 & Os fungos são maléficos* & 295 & 2,84 & \pm & 0,93 \\
\hline
\end{tabular}

* Afirmação conceitualmente incorreta. Legenda: DP: desvio padrão.

Verificando esses resultados de outra forma, dos 295 respondentes, $95(31,1 \%)$ discordaram da afirmativa [1+2 da escala Likert], enquanto $65,6 \%$ concordaram [3+4 da escala Likert] e 3,3\% não opinaram. Os dados evidenciaram uma percepção em que os estudantes, em sua maioria, consideram que os fungos causam mal, o que demonstra uma visão distorcida em relação a este grupo de organismos. Este resultado corrobora fortemente com o ponto de vista defendido por autores de estudos relacionados ao ensino de micologia [10, 11].

Novossate (2011) [12], ao investigar o ensino de micologia entre professores e alunos do Ensino Fundamental, descreveu que:

É preciso propor atividades que permitam reflexões e discussões [...] que possibilitem a ampliação e ressignificação de noções maniqueístas de fungos maus que estão arraigadas tanto entre os estudantes quanto entre os professores 
que colaboraram neste estudo. Algumas dessas noções apresentam os fungos como "coisas" ou seres ruins, negativos ou nojentos [12].

Ao entrecruzar os dados de Novossate (2011) [12] com os desta pesquisa, é possível perceber que esta visão distorcida dos fungos como organismos maléficos já foi evidenciada desde muito antes do estudante chegar ao ensino médio, no caso do trabalho de Novossate (2011) [12], ainda no Ensino Fundamental I. Outro dado preocupante, apontado por esta autora, é que esta visão não foi evidenciada apenas nas crianças, mas também em seus professores, indicando um problema na formação e prática de professores que ensinam os conteúdos de ciências no Ensino Fundamental I.

Sobre este aspecto, Delizoicov et al. (2002) [13] discutem que o entendimento de um professor sobre um conteúdo influencia a maneira de pensar sobre ele e de ensiná-lo. Assim, dificuldades para lidar com um tema podem levá-lo a ignorar ou evitar o conteúdo em sala de aula ou até mesmo influenciar o estudante a ter a mesma visão que do professor sobre um assunto, tirando do aluno a possibilidade de elaboração/reelaboração ou ampliação de sua própria perspectiva [13].

\subsection{Fator "Saúde e Doença"}

A Tabela 2 exibe as médias da percepção e conhecimento dos estudantes em relação as afirmativas do fator "Saúde e Doença".

Tabela 2: Média das afirmações do fator "Saúde e Doença".

\begin{tabular}{|c|c|c|c|c|c|}
\hline Afirmação & Descrição & (n) & Média & & DP \\
\hline A 05 & Os fungos causam doenças em humanos & 289 & 3,17 & \pm & 0,96 \\
\hline A $06^{*}$ & As caspas do couro cabeludo são fungos & 284 & 2,65 & \pm & 1,19 \\
\hline A $11 *$ & As cáries dentárias são causadas por fungos & 286 & 2,53 & \pm & 1,13 \\
\hline A 12* & Os fungos quando se associam a outros seres vivos causam doenças & 288 & 3,09 & \pm & 0,94 \\
\hline A 21 & As frieiras são doenças provocadas por fungos & 286 & 3,27 & \pm & 1,01 \\
\hline A 24 & Os fungos causam as micoses de unha & 288 & 3,47 & \pm & 0,89 \\
\hline A 25 & A candidíase é uma doença causada por fungos & 269 & 2,78 & \pm & 1,11 \\
\hline A 26 & Os fungos podem causar doenças em animais domésticos, como cães e gatos & 275 & 2,68 & \pm & 1,18 \\
\hline
\end{tabular}

* Afirmações conceitualmente incorretas. Legenda: DP - desvio padrão.

Os dados evidenciaram que a maior parte dos estudantes exibe noções adequadas sobre como os fungos acometem a saúde humana. As afirmações conceitualmente corretas deste fator (A 05; A 21; A 24; A 25; A 26) apresentaram médias acima de 2,5, indicando concordância da maioria dos estudantes com as afirmações.

Silva et al. (2009) [14], ao investigarem as concepções prévias de alunos do ensino médio sobre os fungos, antes da realização de uma atividade prática, constataram que parcela significativa dos estudantes pensa em fungos exclusivamente como seres patogênicos. Nesta mesma perspectiva, ideias conceitualmente errôneas relacionadas a este fator, também foram apontadas nesta pesquisa, como é o caso da ideia de que as caspas do couro cabeludo são fungos (A 06), de que as cáries dentárias são causadas por fungos (A 11) e que quando os fungos se associam aos humanos causam doenças (A 12). Esse resultado reforça a ideia de que a concepção dos estudantes em relação aos fungos está fortemente associada a aspectos negativos.

No que tange este assunto, Silva e Menolli Jr (2016) [15], ao analisarem o conteúdo de micologia em livros didáticos de biologia destinados ao ensino médio, constataram que algumas obras apresentam excesso de informações sobre fungos patogênicos em seus textos. Para os autores, a quantidade elevada dessas informações contribui para esta concepção: 
Vale ressaltar que, apesar de ser um tema importante, a quantidade elevada de informações sobre fungos patogênicos pode induzir o aluno a ter ideia de que a principal importância dos fungos está relacionada ao fato de tais organismos serem causadores de doenças humanas, ao passo que apenas representantes de algumas espécies, dentre todas até então conhecidas, são sabidamente patogênicas [15].

\subsection{Fator "Ciência, Tecnologia e Sociedade"}

As médias das afirmações do fator "Ciência, Tecnologia e Sociedade", realçaram dados interessantes (Tabela 3). A afirmação A28 ("Alguns fungos são comestíveis"), por exemplo, foi o único item do fator com resultado acima da média da escala Likert $(\mathrm{A} 30=2,61)$, mesmo assim, a diferença é pequena.

Tabela 3: Média das afirmações do fator "Ciência, Tecnologia e Sociedade”.

\begin{tabular}{|c|c|c|c|c|}
\hline Afirmação & Descrição & (n) & Média & DP \\
\hline A 03 & Os fungos são utilizados na produção do combustível etanol (álcool) & 284 & 1,99 & $\pm 1,21$ \\
\hline A 08 & Os fungos são utilizados na produção de pães & 285 & 2,19 & $\pm 1,32$ \\
\hline A 14 & Fungos são utilizados em biorremediação (descontaminação de ambientes poluídos) & 267 & 2,19 & $\pm 1,16$ \\
\hline A 17 & Os fungos são utilizados na produção de medicamentos & 287 & 2,31 & $\pm 1,25$ \\
\hline A 20 & Os fungos são utilizados na produção de bebidas, como vinhos e cervejas & 284 & 2,26 & $\pm 1,29$ \\
\hline A 28 & Alguns fungos são comestíveis & 288 & 2,61 & $\pm 1,23$ \\
\hline
\end{tabular}

Legenda: DP - desvio padrão.

As demais afirmações apresentaram valores abaixo da média da escala Likert, indicando pouca noção dos estudantes sobre as aplicações e uso dos fungos em processos biotecnológicos relacionados ao cotidiano.

Sobre os possíveis porquês deste resultado, Marques et al. (2016) [11] narraram que, no contato com escolas de ensino fundamental e médio, percebe-se que o estudo dos fungos está se "limitando à memorização de conceitos e terminologias, sem estímulo a questionamentos por parte dos estudantes". Aplicações biotecnológicas dos fungos já consagradas na ciência e sociedade, como é o caso do uso de leveduras na produção de etanol e bebidas fermentadas (A03; A20) não são percepções evidentes na visão dos estudantes. Vale salientar que este assunto está diretamente associado ao conteúdo de produção energética dos seres vivos por fermentação, presente nos currículos de ciências naturais e biologia.

A média da percepção dos estudantes nas afirmações relativas a produção de pães e medicamentos (A08 e A17), outras aplicações conhecidas desses seres em processos biotecnológicos e que estão fortemente atrelados ao cotidiano, também apresentaram valor abaixo da média (A08; A17 <2,5), evidenciando que os estudantes possuem pouco conhecimento sobre o assunto. Resultado semelhante foi obtido na análise da afirmação A14, que questionou aos estudantes sobre o emprego de fungos em processos de biorremediação de ambientes, tópico atrelado ao entendimento sobre o processo de decomposição e ciclagem da matéria na natureza.

Santos et al. (2016) [10], com objetivo de popularizar o conhecimento científico relacionado aos fungos, ministraram uma oficina denominada "A importância dos fungos para sociedade humana", no Museu Espaço Ciência, no município de Olinda/PE. Essa oficina, que teve como público-alvo a população em geral, também investigou o que as pessoas conhecem e/ou sabem das aplicações dos fungos na sociedade e cotidiano. Os autores chegaram à conclusão que as pessoas costumam associar os fungos pontualmente a efeitos negativos e desconsideram as ações benéficas dos fungos para o meio ambiente e para sociedade humana [10]. Os autores também relataram que a maioria das pessoas não reconhece ou consegue exemplificar o uso e aplicações dos fungos no 
dia a dia [10]. Esse relato, junto aos resultados da presente pesquisa, demonstra deficiente alfabetismo científico na sociedade em geral no que tange os fungos.

Esses resultados também tendem a evidenciar que apesar da presença constante da biologia no dia a dia da população, o ensino desta disciplina ainda se encontra distante da realidade, o que não permite à população perceber o vínculo existente entre o que é estudado na disciplina e o cotidiano. De acordo com Moraes (2016) [16], essa visão dicotômica impossibilita ao estudante o estabelecimento de relações entre a produção científica e o seu contexto, prejudicando a necessária visão que deve pautar o aprendizado sobre esta ciência.

\subsection{Fator "Ecologia e Ambiente"}

As médias das percepções e conhecimentos dos estudantes em relação ao fator "Ecologia e Ambiente" são expressas na Tabela 4.

Tabela 4: Média das afirmações do fator "Ecologia e Ambiente".

\begin{tabular}{|c|c|c|c|c|c|}
\hline Afirmação & Descrição & (n) & Média & & DP \\
\hline A $04 *$ & É mais importante preservar as plantas e os animais do que os fungos. & 284 & 2,90 & \pm & 1,11 \\
\hline A 16 & Os fungos são responsáveis, junto às bactérias, pelo processo de decomposição. & 294 & 3,04 & \pm & 1,08 \\
\hline A 22 & $\begin{array}{l}\text { A partir da degradação de matéria orgânica, os fungos enriquecem o solo com } \\
\text { nutrientes. }\end{array}$ & 277 & 2,77 & \pm & 1,12 \\
\hline A 23 & Os fungos provocam doenças em plantas e causam prejuízos na agricultura. & 275 & 2,42 & \pm & 1,15 \\
\hline A 29 & Os fungos auxiliam as plantas na absorção de água e minerais do solo. & 275 & 2,50 & \pm & 1,2 \\
\hline
\end{tabular}

* Afirmações conceitualmente incorretas. Legenda: DP - desvio padrão.

A análise das médias das afirmações A16 e A22 evidenciou que a maioria dos estudantes exibe noção da atuação dos fungos como organismos decompositores, percepção importante para a compreensão dos processos relativos à teia da vida, a ciclagem dos materiais e a manutenção dos ecossistemas (A16; A22 > 2,5). Porém, a afirmação A04 mostrou uma percepção tendendo a concordar que para os estudantes é mais importante preservar/conservar as plantas e os animais do que os fungos (Q6 > 2,5). Ou seja, para a maioria dos estudantes as plantas e os animais têm uma importância no meio ambiente superior à dos fungos. Um dado como este não pode ser ignorado, visto que, na teia da vida, não há uma hierarquia entre os grupos de seres vivos na cadeia ecológica. Nesse sentido, há uma percepção importante a ser trabalhada no processo de construção do conhecimento científico na escola: a ideia de que os fungos são tão importantes quanto qualquer outro grupo de seres vivos e, assim, necessitam ser conservados.

Já os itens A23 e A29 atestam resultados próximos ao valor médio da escala Likert, evidenciando que uma parte dos estudantes propende a concordar com as afirmativas, enquanto outra parte discorda. Vale ressaltar, que ambas as afirmações, se tratam de relações ecológicas entre fungos e plantas. A análise da média da afirmação A23, quando comparada com a afirmação A05 do fator "Saúde e Doença" (A05: Os fungos causam doenças em humanos), indica que os estudantes têm mais percepção de que fungos causam doenças em humanos do que em plantas (A05 3,17 > A23 2,42).

Sobre os conceitos relacionados a este fator, Johan et al. (2014) [17] salientam que, na escola, em virtude da supervalorização dos conteúdos conceituais e descritivos, por vezes a importância ecológica e as relações dos fungos com os demais seres vivos são esquecidas ou pouco consideradas. Já Silva e Menolli Jr (2016) [15], ao analisarem o conteúdo de micologia nos livros didáticos de biologia, chegaram à conclusão de que a abordagem ecológica e ambiental dos fungos é um dos tópicos com maior deficiência conceitual nas obras. 


\subsection{Fator "Biologia dos Fungos"}

A análise dos dados do fator "Biologia dos fungos", apresentados na Tabela 5, também revelou dados importantes da percepção e conhecimento dos estudantes, como a ideia de que fungos são bactérias $(\mathrm{A} 10=3,10)$. Ao olhar os dados percentuais dessa afirmação, verificou-se que $24,0 \%$ dos estudantes, de alguma forma, discordaram [1+2] do item A10, enquanto $72,1 \%$ concordaram [3+4] e outros 3,9\% não opinaram. Assim, aparentemente, um pouco mais da metade dos estudantes não distinguem claramente fungos de bactérias. Inclusive, a maioria dos estudantes também apontou que os fungos são procariontes $(\mathrm{A} 02=2,81)$, característica específica de bactérias.

Tabela 5: Média das afirmações do fator "Biologia dos Fungos".

\begin{tabular}{|c|c|c|c|c|}
\hline Afirmação & Descrição & (n) & Média & DP \\
\hline A 02* & $\begin{array}{l}\text { Os fungos são procariontes (o material genético não é delimitado por } \\
\text { membrana nuclear) }\end{array}$ & 269 & 2,81 & 1,16 \\
\hline A $07 *$ & Os fungos são organismos menos evoluídos que os animais & 277 & 2,92 & 1,11 \\
\hline A 09* & $\begin{array}{l}\text { Os fungos são encontrados apenas no ambiente terrestre (não existem } \\
\text { fungos nos rios ou mares) }\end{array}$ & 286 & 2,35 & 1,28 \\
\hline A $10 *$ & Alguns fungos são bactérias & 293 & 3,10 & 1,16 \\
\hline A $13 *$ & Os fungos apresentam parede celular constituída de celulose & 264 & 2,82 & 1,12 \\
\hline A $15 *$ & Mofos e bolores não são vivos & 288 & 2,39 & 1,28 \\
\hline A $18 *$ & Os fungos realizam fotossíntese & 286 & 2,57 & 1,25 \\
\hline A $19 *$ & Os fungos são considerados plantas & 290 & 2,22 & 1,24 \\
\hline A 27 & Os fungos alimentam-se por absorção de nutrientes & 275 & 2,80 & 1,10 \\
\hline
\end{tabular}

* Afirmações conceitualmente incorretas.

Ainda nesta perspectiva, vale destacar os trabalhos que também discutiram esta questão em suas pesquisas. Silva (2019) [4], ao estudar a visão dos estudantes sobre fungos, solicitou que estes descrevessem as primeiras palavras que lhe viessem a lembrança quando pensassem em fungos. $\mathrm{O}$ resultado foi que mais de um terço dos estudantes investigados apontaram a palavra bactéria (ou termos semelhantes) como associada aos fungos [4]. Já Novossate (2011) [12], investigou a percepção de professores, contudo, o resultado não foi muito diferente. A autora relata que "alguns confundiram fungos com bactérias como se os dois pertencessem ao mesmo grupo, ou como sendo os mesmos tipos de seres vivos" [12].

Outro erro conceitual que aparentemente compõe a percepção de parte dos estudantes é a ideia de que fungos apresentam parede celular constituída de celulose $(\mathrm{A} 13=2,82)$ e que realizam fotossíntese (A18 $=2,57)$, características ausentes em tais organismos. Em contrapartida, no que se refere à afirmação de que fungos são considerados plantas $(\mathrm{A} 19=2,22)$, os estudantes exibiram média abaixo de 2,5, indicando que a maioria dos estudantes percebe que plantas e fungos pertencem a grupos de seres vivos distintos.

Já a afirmação A07 deste fator exprimiu uma visão distorcida dos estudantes quanto à ideia de evolução biológica, em especial à evolução dos fungos. A média geral desta afirmação foi de 2,92, indicando concordância dos respondentes quanto à questão de que os fungos são menos evoluídos que os animais. Essa visão distorcida sobre a evolução biológica já foi discutida por outros autores do ensino de ciências e biologia, como Paiva (2015) [18], que relatou que essa ideia errônea de compreender determinados organismos como mais evoluídos que outros é uma visão bastante comum, uma tendência gerada devido aos alunos comumente associarem evolução biológica ao aumento de complexidade, crescimento e melhora do ser vivo.

Paiva (2015) [18] investigou 36 estudantes, também concluintes do $3^{\circ}$ ano do ensino médio, quanto à seguinte questão: "Um elefante é mais evoluído que uma bactéria?". Como previsto pelo autor, um grande grupo de alunos associou evolução biológica à complexidade, considerando o elefante mais evoluído que a bactéria. 
Dessa forma, os resultados desta pesquisa reforçam a ideia de como o entendimento sobre a evolução biológica ainda é confuso e equivocado na compreensão de parte das pessoas.

Quanto ao processo de alimentação dos fungos, investigado na afirmação A27, os estudantes mostraram uma percepção e conhecimento com valor médio de 2,80, indicando concordância de que os fungos se alimentam por absorção, uma concepção conceitualmente correta. Já as afirmações A09 e A15, indicaram tendência à discordância, demostrando que a maioria dos estudantes percebe que os fungos, além dos ambientes terrestres, são encontrados em ambientes aquáticos e que mofos e bolores tratam-se de espécies de fungos.

\section{CONCLUSÃo}

Esta pesquisa revelou importantes percepções e conhecimentos que estudantes concluintes do ensino médio possuem sobre os fungos quando estão prestes a finalizar a educação básica. Os resultados evidenciaram um notório analfabetismo científico deste público em relação aos conteúdos micológicos. Os estudantes percebem e conhecem mais os fungos como organismos patogênicos do que seres vivos com fundamental importância ecológica na teia da vida ou que se destacam em processos biotecnológicos relacionados a vida cotidiana.

Há muitos conceitos equivocados sobre os fungos na visão dos estudantes, entre eles a ideia de que são biologicamente menos evoluídos, que apresentam importância ecológica inferior a outros seres, que são organismos maléficos ou então são confundidos com bactérias. Esses resultados levam a considerar que parte dos estudantes deixam a escola básica com conhecimentos limitados sobre os fungos, de forma a pouco perceber esses organismos no cotidiano e no meio ambiente.

Wandersee e Schussler (1999) [19], numa perspectiva semelhante, propuseram o termo "cegueira botânica" para se referir à falta de habilidade das pessoas em perceber a importância das plantas para o planeta. Talvez seria necessário se pensar se também não existe uma "cegueira micológica", pois como evidenciado neste estudo, de modo geral, as pessoas pouco conhecem sobre os fungos.

Por fim, cabe acentuar que o ensino de fungos carece de atenção. É necessário o desenvolvimento de pesquisas que ampliem e fundamentem os debates sobre o ensino de micologia nas escolas e nas instituições de formação de professores. Espera-se que os resultados desta pesquisa contribuam com reflexões que possam impactar positivamente no fazer pedagógico dos professores e, consequentemente, no aprender dos estudantes, em especial, quando o assunto em questão forem os fungos.

\section{REFERÊNCIAS BIBLIOGRÁFICAS}

1. Raven PH, Evert RF, Eichhorn SE. Biologia Vegetal. 8. ed. Rio de Janeiro: Guanabara Koogan; 2014.

2. Putzke J, Putzke MTL. O Reino dos Fungos. 2. ed. Santa Cruz do Sul (RS): EDUNISC; 2004.

3. Silva BA, Bastos SND. Ensino aprendizagem de micologia no ensino médio: um estudo de caso na EEEFM Padre Luiz Gonzaga, Bragança, Pará. In: Anais da 62a Reunião Anual da Sociedade Brasileira para o Progresso da Ciência; 25-30 jul 2010; Natal (RN). Natal (RN): SBPC; 2010.

4. Silva AC. A visão dos alunos sobre fungos: estudo das percepções e conhecimentos de fungos por estudantes concluintes do ensino médio [dissertação]. Diadema (SP): Universidade Federal de São Paulo; 2019. $129 \mathrm{p}$.

5. Sena BL, Santos SX. Estudando fungos por meio de atividades práticas. In: Neves MA, Giachini AJ, editores. Anais do $8^{\circ}$ Congresso Brasileiro de Micologia; 03-06 out 2016; Florianópolis (SC). Florianópolis (SC): Sociedade Brasileira de Micologia; 2016.

6. Vieira KM, Dalmoro M. Dilemas na construção de escalas tipo Likert: o número de itens e a disposição influenciam os resultados? Rev Gestão Organizacional. 2013;6(3):162-174.

7. Severino AJ. Metodologia do trabalho científico. 23. ed. São Paulo: Cortez; 2007.

8. Marotti J, Galhardo APM, Furuyama RJ, Pigozzo MN, Campos TN, Laganá, DC. Amostragem em Pesquisa Clínica: tamanho da amostra. Rev Odontologia Univ Cidade São Paulo. 2008;20(2):186-194.

9. Gerhardt TE, Silveira, DT, organizadores. Métodos de pesquisa. Porto Alegre (RS): Editora da UFRGS; 2009.

10. Santos JEF, Silva EM, Silva BWC, Santos AQ, Silva RNM, Bezerra W, Carvalho DA. Popularização da Ciência: fungos, a sociedade humana e o meio ambiente. In: Neves MA, Giachini AJ, editores. Anais do 
$8^{\circ}$ Congresso Brasileiro de Micologia; 03-06 out 2016; Florianópolis (SC). Florianópolis (SC): Sociedade Brasileira de Micologia; 2016.

11. Marques MFO, Moraes TS, Carvalho FLQ. Percepção dos estudantes da educação básica frente à utilização de jogos educativos na abordagem CTS. In: Anais da $11^{a}$ Jornadas Latino-americanas de Estudos Sociais da Ciência e Tecnologia; 25-28 jul 2016; Curitiba (PR). Curitiba (PR): Associação Latino-americana de Estudos Sociais da Ciência e da Tecnologia; 2016.

12. Novossate S. Uma bola de neve rolou para o Vale do Ribeira: nela havia cogumelos, professores, livros, crianças (além de duendes e sacis). Contexto \& Educação. 2011;26(86):74-101, doi: 10.21527/21791309.2011.86.74-101

13. Delizoicov D, Angotti JA, Pernambuco MM. Ensino de Ciências: fundamentos e métodos. São Paulo: Cortez; 2002.

14. Silva JC, Macêdo PB, Coutinho A C, Silva CH, Rodrigues CWMS, Oliveira GF, Araújo ML F. Estudando fungos a partir de uma prática problematizadora e dialógica: relato de uma experiência no ensino médio em uma escola pública. In: Anais da 9 Jornada de Ensino Pesquisa e Extensão; 2009; Recife (PE). Recife (PE): Universidade Federal Rural de Pernambuco; 2009.

15. Silva AC, Menolli Jr N. Análise do conteúdo de fungos nos livros didáticos de Biologia do ensino médio. Ciência \& Ideias. 2016;7(3):235-273, doi: 10.22407/issn.2176-1477.2016v7i3619

16. Moraes TS. Estratégias inovadoras no uso de recursos didáticos para o Ensino de Ciências e Biologia [dissertação]. Salvador (BA): Universidade do Estado da Bahia; 2016. 144 p.

17. Johan CS, Carvalho MS, Zanovello R; Oliveira RP, Garlet TMB, Barbosa NBV, Moresco TR. Promovendo a aprendizagem sobre fungos por meio de atividades práticas. Ciência Natura. 2014;36 (Ed. Especial II):798-805, doi: 10.5902/2179460X12607

18. Paiva TMA. Concepções de estudantes concluintes do ensino médio acerca da evolução biológica. In: Anais do $2^{\circ}$ Congresso Nacional de Educação; $14-17$ out 2015; Campina Grande (PB). Campina Grande (PB): Realize Editora; 2015.

19. Wandersee JH, Schussler, EE. Preventing plant blindness. Am Biol Teacher. 1999;61(2):284-286, doi: $10.2307 / 4450624$ 